Sādhanā Vol. 36, Part 4, August 2011, pp. 425-440. (C) Indian Academy of Sciences

\title{
Application of a stochastic modelling framework to characterize the influence of different oxide scales on the solid particle erosion behaviour of boiler grade steel
}

\author{
S K DAS \\ National Metallurgical Laboratory, Council of Scientific and Industrial Research, \\ Jamshedpur 831007, India \\ e-mail: skd@nmlindia.org
}

MS received 1 November 2010; revised 28 March 2011; accepted 20 May 2011

\begin{abstract}
An earlier developed stochastic model has been applied to describe the relative rate of material loss from the steel surface subjected to simultaneous action of high temperature oxidation involving multiple oxides and mechanical erosion. Different oxide scale growths, namely, nickel, iron and chromium have been treated deterministically and erosion is described using a literature based probabilistic framework. Oxidation is described with a power law (parabolic) approach to quantify the rate of growth of all the three oxide scales. In consonance with the published model, erosion is treated using a probabilistic methodology as spatially random phenomena on the oxide surface. The concept of 'erosion footprint' has been incorporated in the present model to characterize the erosion-induced damage on the steel surface. The model has been applied to predict the relative material loss as a function of time resulted from erosion-oxidation interaction pertaining to nickel, iron and chromium oxides in dimensionless form. This investigation is expected to provide a quantitative understanding of relative material loss due to solid particle erosion for oxide scales, (composed of multiple oxides) formed on the steel components of coal-fired boilers.
\end{abstract}

Keywords. Stochastic modelling; erosion-oxidation interaction; oxidation kinetics; erosion footprint; scale thickness; gamma function.

\section{Introduction}

Degradation due to high temperature erosion by fly ash particles is a serious issue with regard to life cycle management of coal-fired power plants. The erosion of steel components operating at elevated temperatures is also subjected to simultaneous formation of surface scales such as oxides. High temperature erosion, which involves the conjoint action of high temperature oxidation and damage due to solid particle impact, is a complex process. Material damage occurs through the interaction of both oxidation and erosion, with metal recession rates often enhanced as a result of the synergetic effect between the two processes. There are occasions when oxidation can reduce the degree of materials loss by such wear processes by forming a stable protective 
oxide layer, over the metallic substrate often referred to as an oxide glaze, but more often than not the combination results in excessive high wear rates. Figure 1 shows the schematic of a coal-fired power plant and its components.

Oxidation is a thermo-chemical process. In general, all metals when exposed at high temperature move towards a thermodynamic equilibrium with their environment. In steel/alloy systems, there is competition between various alloy constituent, which give rise to selective oxidation. Simultaneous growth of these species usually leads to the formation of a multi-layered scale in the form of a protective oxide on the metallic surface, be it alumina, chromia or iron oxide, depending on the alloy being oxidized. Oxidation kinetics is of significant importance in terms of determining the rate of growth of various oxides. In general, Nickel oxide (NiO) has a relatively faster growth rate, where as chromium oxide $\left(\mathrm{Cr}_{2} \mathrm{O}_{3}\right)$ growth rate is slower and iron oxide $(\mathrm{FeO})$ has an intermediary rate of growth. When metal is subjected to erosion and oxidation simultaneously at high temperature, the metal surface exhibit certain topographical features that reflect the nature of the wear and the conditions under which it occurs. There are two distinct primary regions of erosion-oxidation interaction. In the initial stage, the erosion removes the surface of the growing oxide scale without affecting the metal-oxide scale interface or the metal below. In this regime, the eroded oxide surface shows evidence of cutting wear, removal and displacement that reflect various degrees of plastic displacement or removal of the oxide. The oxide scale surface remains relatively flat and does not develop any other topographical features. In the second primary regime of interaction, in which the erosion component is substantially more vigorous, the distinct oxide scale no longer forms and the erosive particles impact the metal

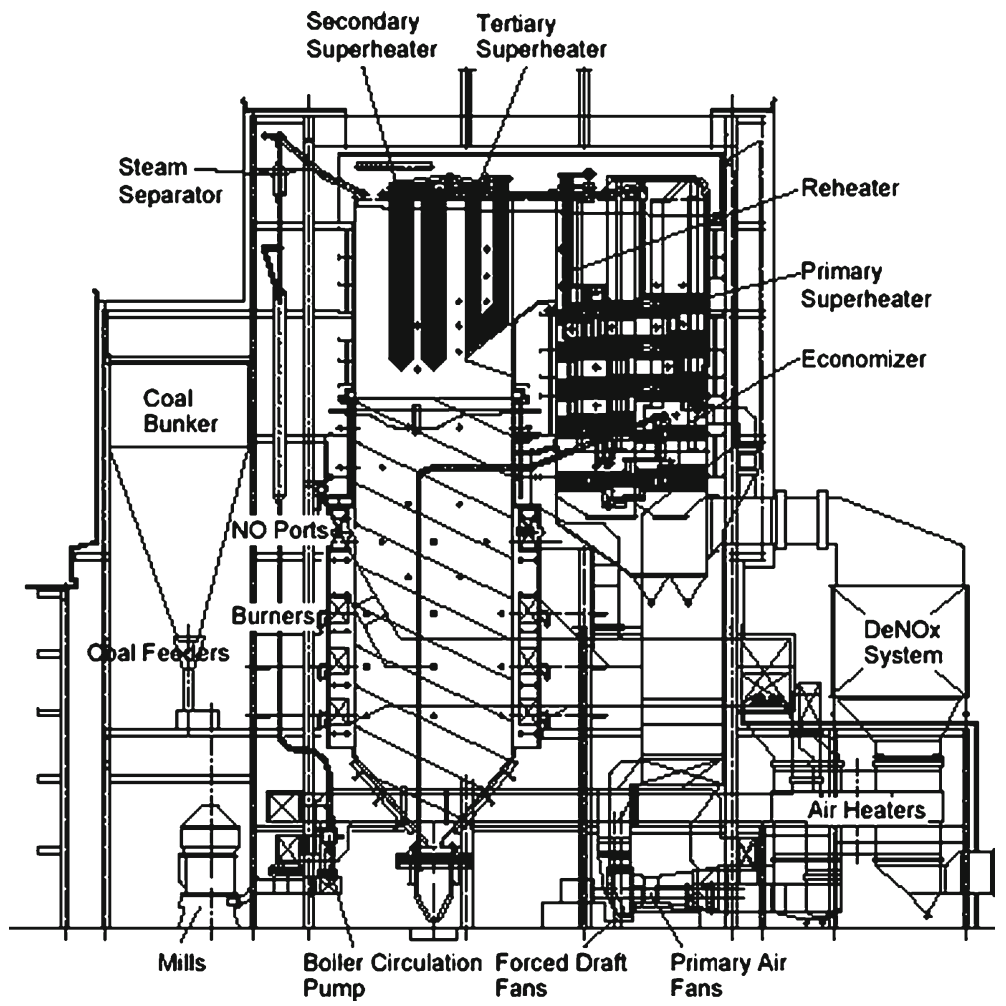

Figure 1. Schematic of a coal fired super-thermal power plant. 
substrate, causing substantial plastic deformation of the metal. Description of the phenomena of erosion-oxidation in a way that makes use of the full breadth of understanding gained from studies of each phenomena operating separately, is particularly difficult and challenging because of the generally complex nature of both processes and the many potential combinations of the two phenomena.

This paper attempts to describe the erosion-oxidation response and behaviour of boiler grade steel in a high temperature-oxidizing environment incorporating a previously developed probabilistic modelling framework (Markworth 1992; Wright et al 1995; Nicholls \& Stephenson 1995). An attempt has been made to quantify the relative material loss as a function of time resulted from erosion-oxidation interaction pertaining to nickel, iron and chromium oxides using dimensional analysis and probabilistic model. In order to make the problem mathematically tractable, the present formulation incorporates a simplifying assumption that kinetics of formation of different oxide scales, namely, $\mathrm{NiO}, \mathrm{FeO}$ and $\mathrm{Cr}_{2} \mathrm{O}_{3}$ independent of each other and growth of one oxide is not influenced by the other (i.e., non-coupled growth kinetics).

\section{Damage mechanisms of erosion-oxidation process}

The erosion of metals operating at elevated temperatures will be influenced by the formation of surface oxide scales. Based on the tensile properties, the metal substrate will respond in a ductile manner whereas the oxide scale may exhibit a brittle response. Thus, at high temperature erosion can be considered as a phenomenon of material removal from a composite system, consisting of a ductile substrate and brittle surface oxide. Mechanisms of removal are attributed to oxide fracture, chipping, and oxide spallation at the metal-oxide interface and plastic deformation of metal substrate resulting in both oxide and metal loss.

For low energy impacting particles, scale fracture does not occur and therefore metal recession rates will be determined by only oxidation process. As the impact energy is increased and damage to the oxide scale occurs, an interaction between erosion and oxidation will result in acceleration of metal loss. When a metal undergoing oxidation is subjected, simultaneously, to a flux of erosive particles, the surface of the growing oxide layer will be eroded at a rate that will be proportional to the potency of the erosive flux. Under conditions such that a scale of substantial thickness if formed, the removal of oxide from scale surface is reflected from the observed topography and surface characteristics. These features include the well-known cutting, plowing, cracking and indention marks that reflect the processes by which oxide is removed from the scale surface, as well as various degrees of plastic deformation that accompany the processes (Finnie 1960; Bitter 1963a,b; Shewmon 1981). The surface features mentioned above are caused by the erosive impacts, which also give rise to a zone of compression immediately below the impact site. Under the compression, the substrate will tend to deform to an extent determined by the hardness and tensile properties of the oxide scale observed so far, the surface remains flat and does not develop other, larger scale, topographical features. This indicates that immediately subsurface of the oxide is not deformed plastically under the impacts to any substantial depth. With more vigorous erosion, compared with the rate of oxidation, a distinct scale is no longer able to form and the incident particles impact directly onto the metal substrate. The particles penetrate more deeply into the comparatively ductile metal substrate. There are some critical factors, which influence the erosion-oxidation phenomena of metallic materials. These include, particle size, velocity, density, angularity, hardness, particle flux concentration, angle of impact and the oxidation kinetics, which are influenced by the temperature and alloy composition. Characterization and quantification of material loss (Stack \& Pena 2001; Hogmark et al 1983) as a 
consequence of erosion-oxidation interaction on a metallic surface, a priori information on the following is necessary.

- The oxidation properties of the substrate (metal).

- The erosion properties of the substrate.

- The erosion properties of the oxide layer.

- The erosion properties of the substrate coated with an oxide layer.

The oxidation properties of the base metal in conjunction with the flux of the erodent will determine the thickness of the oxide growth. The velocity and size of the particles will also have a significant influence on the effective scale growth rate.

The erosion properties of the oxide layer assume importance when the impact energy and the particle flux are both sufficiently low. Consequently, the oxide grows to a sufficient thickness that the impact event is predominantly confined to the oxide layer and is not affected by the substrate. The erosion properties of the base layer with an oxide scale become important when the oxide layer is not sufficiently thick to absorb the particle impact energy resulting in deformation of both oxide scale and substrate concurrently. The combination of these different factors necessitates definition of various erosion regimes/mechanisms incorporating factors such as: particle size, flux, velocity and oxide/substrate interactions.

In general, erosion-oxidation (Chang et al 1990; Stephenson et al 1985, 1986; Stephenson \& Nicholls 1990) process has been characterized into four different regimes ranging from pure erosion on one side to pure oxidation on the other with various intermediate regimes. Figure 2 shows a schematic of various regimes of erosion-oxidation interaction mechanism. In the oxidation regime (regime-I), only oxide scale formation takes place as per the growth kinetics. In the erosion-modified regime (regime-II), the erosive components remove only the outer part of a continuous and growing oxide layer and effectively reduces the thickness of the oxide scale, leading to a more rapid rate of oxidation than expected. The oxidation rate is controlled by the diffusion across the oxide layer. In the oxidation modified erosion regime (regime-III), the rate of oxide formation is slow such that the oxide removal makes a negligible contribution to the overall material loss. The erosion is predominantly attributed to the removal of metal. In the metallic erosion regime (regime-IV), the metallic substrate erosion takes place, after the complete removal of the oxide layer. This regime is governed by ductile erosion of the metal.

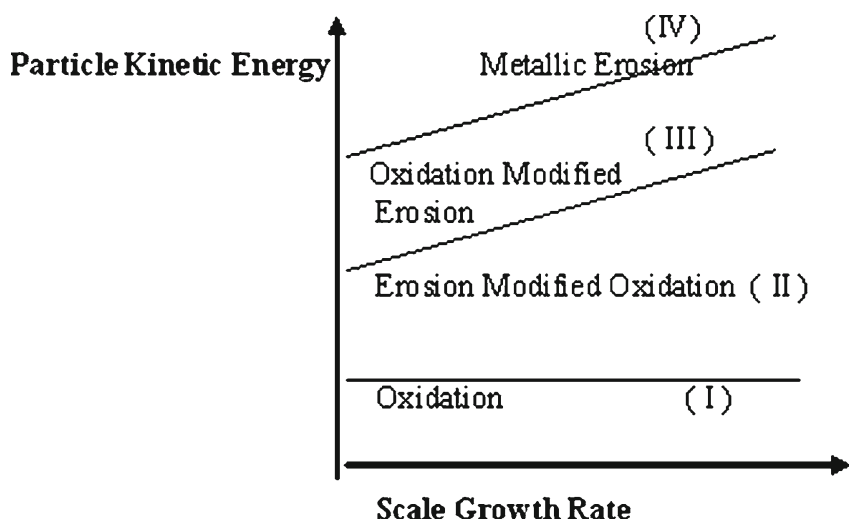

Figure 2. Schematic diagram showing oxidation-erosion interaction mechanism. 


\subsection{Modelling concepts}

From the viewpoint of quantitative modelling of erosion-oxidation phenomena, it is reported by Stack \& Scott (1993) that, there are two main methodologies that have been followed. The first involves 'one equation' approach where the oxidation kinetics of a system are combined with simple mass loss equations to generate a single complex equation, which can predict mass loss because of wear generated through combined action of erosion-oxidation. This type of model has a limited accuracy and is normally valid for only one specified mechanism. This model was subsequently improved by Stack \& Scott (1993) with the introduction of a conceptual area 'erodent contact footprint', which is characterized by the assumption that the volume of oxide completely removed within the footprint circular area after the impact. Footprint size was based on experimental observations and the quantitative methodology is based on a single mass loss equation.

Another more complex formalism that was attempted by Wellman \& Nicholls (2004) pertained to the use of Monte-Carlo simulation, where the damage caused by each individual impacting particle is quantified. The erosion behaviour of a ceramic-coated surface governed by elasto-plastic interactions between the impacting particle and the target surface has been studied using Monte-Carlo simulation. The Monte-Carlo computer model calculates the contact radius for an infinite thickness $\left(r_{\infty}\right)$, which is given by the following equation:

$$
\mathrm{r}_{\infty}=\frac{15\left(m K c^{\prime} R^{2}\right)^{1 / 5} V \sin \alpha^{2 / 5}}{16}
$$

where, $\mathrm{m}, \mathrm{R}$ and $\mathrm{V} \sin \alpha$ are the mass, radius and normal component of the velocity for the particle impacting the surface and

$$
K c^{\prime}=\frac{1}{E c^{\prime}}=\frac{1-v_{p}^{2}}{E p}+\frac{1-v_{c}^{2}}{E c},
$$

where $E c^{\prime}$ is equivalent modulus of the coated surface. $E_{p}$ and $E_{c}$ are respective modules contributions from plastic deformation and cutting mechanisms. $v_{p}$ and $v_{c}$ are volume of material removed as a consequence of plastic deformation and cutting mechanism, respectively.

Although Monte-Carlo approach is complex, this enables the model to assess each particle/oxide/substrate interaction and ascertain the erosion-oxidation regime to determine the amount of material removed as a function of the local impact conditions. In order to model erosion-oxidation phenomena using Monte-Carlo technique, several parametric information need to be known a priori, such as substrate mechanical properties as a function of temperature, impact dynamics, scale composition and its mechanical properties, scale thickness and rate of scale growth.

\section{Modelling methodologies}

\subsection{Power law oxidation model for scale growth}

A simple deterministic approach has been pursued similar to that of Markworth (1992) to address the oxidation phenomena in the absence of any erosion events. The oxide scale thickness $\delta$ is considered to vary with time according to power law dependence. Thickness of the oxide layer that would form during time ' $\theta$ ' is assumed to be given by the following:

$$
\delta=K \theta^{n}
$$


with associated conditions; i.e., at $\theta=0, \delta=0$ and where $K$ and $n$ are mechanism dependent kinetic parameters. $K$ generally being related to temperature, through Arrhenius relationship:

$$
K=A e^{(-Q / R T)}
$$

where, $A$ is an experimentally determined Arrhenius constant (pre-exponential factor), $Q$ is the activation energy, $R$ is the universal gas constant and $T$ is the absolute temperature. For the case of parabolic oxidation, $n=1 / 2$ and $K=K_{\text {para }}$ while for linear oxidation, $n=1$, and $K=K_{\text {Lin }}$. $K_{\text {para }}$ and $K_{\text {lin }}$ are rate parameters for parabolic and linear oxidation kinetics respectively. In the present oxidation model, parabolic scale growth kinetics has been considered. The deterministic analytical description presented here for parabolic kinetics considers the superficial oxide scales as formed by a succession of diffusion layers of oxides.

Erosion can be described as a stochastic phenomenon. In the spatial domain, individual erosion events are assumed to take place in a random manner. It may be further assumed that instantaneous number of events on the entire surface can be assumed to increase deterministically, as a linear function of time. The average erodent flux, ' $F$ ', can thus be defined as the average number of impacts occurring on the surface per unit area per unit time. Each erosion event has been associated with a point of impact, which defines the location of that event. It is further assumed that because of the randomness of the particle impact, the points of impact have been completely de-linked with one another. Therefore, in principle, these events constitute a spatially random distribution of points on the oxide surface at any instantaneous time.

Each erosion event has assumed to leave a characteristic 'footprint' (Markworth 1992; Wright et al 1995) i.e., an area of damage on the surface around its point of impact. This footprint is usually chosen on the basis of experimental investigations. For simplistic analysis, a simple footprint concept can be employed, such as a circle with radius ' $\sigma$ ', representing the area of damage with the point of impact located at the center of the circle. Irrespective of the scale thickness it is assumed that all the oxides within this circle is removed by the particle impact. After the removal of the scale, oxide growth commences again on the metal surface. The details of the concepts of erosion 'footprint' are described elsewhere Markworth (1992). The probabilistic approach which considers a single arbitrarily chosen point as a point of impact of particle. It is perceived that the behaviour predicted for this point would therefore be applicable to the entire surface in a statistically average manner to quantify the overall wear phenomena.

\subsection{Stochastic approach for erosion-oxidation interaction mechanism}

Incorporating the formalism similar to Markworth (1992) model, the points of impact of erodent particles are assumed to be randomly distributed on the oxide surface, and their spatial distribution can be described in terms of Poisson distribution. In terms of probability distribution, $P_{1}(N, F \Omega t)$, of having $\mathrm{N}$ points of impact occurring on an arbitrarily selected area ' $\Omega$ ' of the surface during an time interval ' $\theta$ ' is given by:

$$
P_{1}(N, F \Omega \tau)=\frac{(F \Omega \tau)^{n} \exp (-F \Omega \tau)}{N !},
$$

where, $F$ is the average flux of erodent particle. The parameter ' $F \Omega \tau$ ', which appears as an argument in eqn. (5) is the average number of impacts occurring on area ' $\Omega$ ' in time interval ' $\theta$ '. It may be assumed that both oxidation and erosion commence at time $\theta=0$. This indicates the instantaneous time at which the metal surface is just exposed to oxidation, i.e., $\delta=0$ everywhere 
on the surface and $\delta$ is the oxide scale thickness as the most recent erosion events, prior to time t, that occurred within radius ' $\sigma$ ' of point of impact 'p', occurred within the interval $\theta$ ' to $\theta$ ' $+\mathrm{d} \theta$ ', where $0 \leq \theta^{\prime}<\theta$. This can be represented as a product of the probability that no event occurred on this area during the interval $\theta+\mathrm{d} \theta$ ' to ' $\theta$ ' and probability that at least that one event did occur during the interval ' $\theta$ ' to $\theta$ ' + ' $\mathrm{d} \theta$ '. This can be expressed using Markworth probability approach as:

$$
P_{2}\left(\theta, \theta^{\prime}\right) d \theta^{\prime}=P_{1}\left(0, F \pi \sigma^{2}\left(\theta-\theta^{\prime}-d \theta^{\prime}\right) \times\left[1-P_{1}\left(0, F \pi \sigma^{2} d \theta^{\prime}\right)\right] .\right.
$$

In the above equation, ' $F \pi \sigma^{2} \theta$ ' represents dimensionless time, which will be used as the temporal variable in further analysis.

After appropriate simplification, the expression for the average scale thickness $\langle\delta>$ is represented as:

$$
\langle\delta\rangle=\frac{K}{\left(F \pi \sigma^{2}\right)^{n}}\left(\gamma\left(n+1, F \pi \sigma^{2} \theta\right)+\left(F \pi \sigma^{2} \theta\right)^{n} \exp \left(-F \pi \sigma^{2} \theta\right) .\right.
$$

In the above expression ' $\gamma$ ' is the incomplete gamma function, defined as

$$
\gamma(\xi, x)=\int_{0}^{x} u^{\xi-1} \exp (-u) d u
$$

The equation (8) can further be integrated and the result can be utilized to simplify eqn. (7) in the following manner:

$$
\langle\delta\rangle=\frac{n K}{\left(F \pi \sigma^{2}\right)^{n}} \gamma\left(n, F \pi \sigma^{2} \theta\right) .
$$

\section{2a Kinetic conditions of scale growth:}

(i) Initial growth

- As $F \pi \sigma^{2} \theta \rightarrow 0$, the above equation simplifies to

$$
\langle\delta\rangle_{\text {int }} \rightarrow K \theta^{n}
$$

Under this limiting condition, the kinetics of process consists of pure oxidation.

(ii) Asymptotic steady state growth

- As $F \pi \sigma^{2} \theta \rightarrow \infty$

$$
\langle\delta\rangle_{\text {asy }} \rightarrow \frac{n K}{\left(F \pi \sigma^{2}\right)^{n}} \Gamma(n),
$$

where, $\Gamma(n)$, is the gamma function. The above expression shows that $<\delta>$ is independent of time under this limiting condition. Asymptotic value of $<\delta>$ can be obtained from the above 
expression, considering a zero scale thickness initially. The dimensionless scale thickness $<\delta>^{*}$, which will be calculated as a function of dimensionless time, is defined as follows:

$$
\left.<\delta>^{*}=<\delta>\left(4 F \pi \sigma^{2}\right)^{1 / 2}\right) / K .
$$

\subsection{Erosion-oxidation based damage quantification}

The average total material eroded $<M_{e r}(\theta)>$ per unit area at time ' $\theta$ ' is given by (Markworth 1992; Wright et al 1995):

$$
<M_{e r}(\theta)>=F \pi \sigma^{2} \rho_{0} \int_{0}^{\theta}\langle\delta\rangle d \theta .
$$

The average growth of total mass per unit area $\left\langle M_{\text {oxd }}(\theta)\right\rangle$ in the oxide scale at time ' $\theta$ ' is given as,

$$
<M_{o x_{d}}(\theta)>=\rho_{0}\langle\delta\rangle .
$$

The total erosion loss $\Delta M(\theta)$ per unit area as of time ' $\theta$ ', can be calculated under the combined effects of oxidation and erosion, using the following expression:

$$
\Delta M(\theta)=\left(1-f_{m}\right) M_{0_{x d}}(\theta)-f_{m} M_{e r}(\theta),
$$

where ' $f_{m}$ ' is the mass fraction of oxide associated with the steel/alloy. The first and second terms in the above equation shows the instantaneous mass gain due to oxidation, and instantaneous mass depletion as a result of erosion.

Equations (13) and (14) along with eqn. (15) can be utilized to derive the following equation.

$$
\Delta M(\theta)=\left(1-f_{m}\right) \rho_{0}\langle\delta\rangle-f_{m} F \pi \sigma^{2} \rho_{0} \int_{0}^{\theta}\langle\delta\rangle d \theta .
$$

Equation (16) needs to be solved numerically. Under the limiting conditions as discussed earlier, the following estimates can be obtained.

\subsection{Kinetic conditions for erosion loss}

(i) Initial erosion loss

- As dimensionless time $\left(F \pi \sigma^{2} \theta\right) \rightarrow 0$,

$$
\Delta M(\theta)_{\mathrm{int}} \rightarrow\left(1-f_{m}\right) \rho_{0} K \theta^{n} .
$$

(ii) Asymptotic erosion loss

- As dimensionless time $\left(F \pi \sigma^{2} \theta\right) \rightarrow \infty$

$$
\Delta M(\theta)_{a s y} \rightarrow \Gamma(n) f_{m} \rho_{0}\left(F \pi \sigma^{2}\right)^{1-n} n K \theta .
$$


The asymptotic value of $\langle\delta>$ has been used for the calculation of asymptotic erosion loss. At intervals of $\theta_{0}$ (where, $\theta_{0}=\left(F \pi \sigma^{2}\right)^{-1}$ ), the average cumulative mass loss per unit area, at any instantaneous dimensionless time ' $\theta$,' can be approximately given as

$$
<\Delta M(\theta)_{c u m}>=f_{m} \rho_{0}\left(F \pi \sigma^{2}\right)^{1-n} K \theta .
$$

To make the analysis independent of dimensional variables, dimensionless mass change variable is defined in the following dimensionless form:

$$
\Delta M *\left(F \pi \sigma^{2} \theta\right)=10^{6}\left(4 F \pi \sigma^{2}\right)^{1 / 2} / \rho_{0} A .
$$

\subsection{Numerical method and code development}

The present mathematical model has been implemented in a Visual C++ code, designated as Erosion Simulation Code-3 (EROSIM-3), to solve the governing equations for predicting the oxide scale thickness in dimensionless form. In addition, dimensionless mass loss per unit area has been computed as a function of dimensionless time, for various mass fraction of oxide associated with metallic constituents at different temperature levels. Appropriate numerical routines have been used, to compute the gamma function values in the equations (7), (8), (9) and (11). A Graphical User Interface (GUI), has also been created with the code for user interaction.

The oxidation kinetics parameters used are from the literature (Wright et al 1995) pertaining to iron oxide $\left(\mathrm{Fe}_{2} \mathrm{O}_{3}\right)$; for the form of oxidation rate description used in equation (4), and the activation energy is $86 \mathrm{KJ} / \mathrm{mol}$. The data (Wright et al 1995) pertaining to average erodent flux (F) and footprint size $(\sigma)$, at baseline conditions of $\mathrm{T}=673^{\circ} \mathrm{K}$ are also incorporated. The value of densities of nickel oxide, iron oxide and chromium oxide have been taken as $5210 \mathrm{~kg} / \mathrm{m}^{3}$, $5700 \mathrm{~kg} / \mathrm{m}^{3}$ and $6700 \mathrm{~kg} / \mathrm{m}^{3}$ respectively.

\section{Results and discussion}

Dimensionless variables have been defined for depicting the quantitative behaviour of erosionoxidation phenomena to transform the variables independent of scale. Figure 3 shows the parabolic oxidation kinetics $(n=1 / 2)$ and scale growth behaviour as a function of time, in dimensionless form. The scale growth variation for all oxides as a function of time shows a monotonic

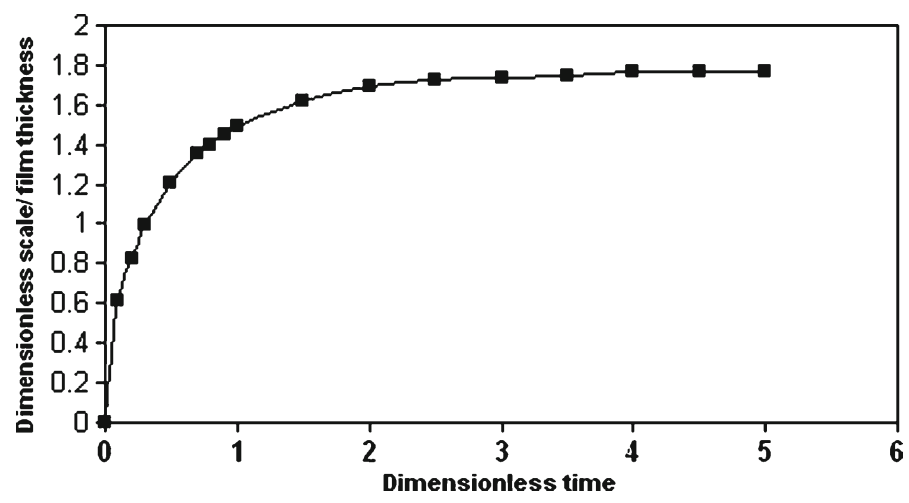

Figure 3. Parabolic oxidation kinetics and scale growth rate. 


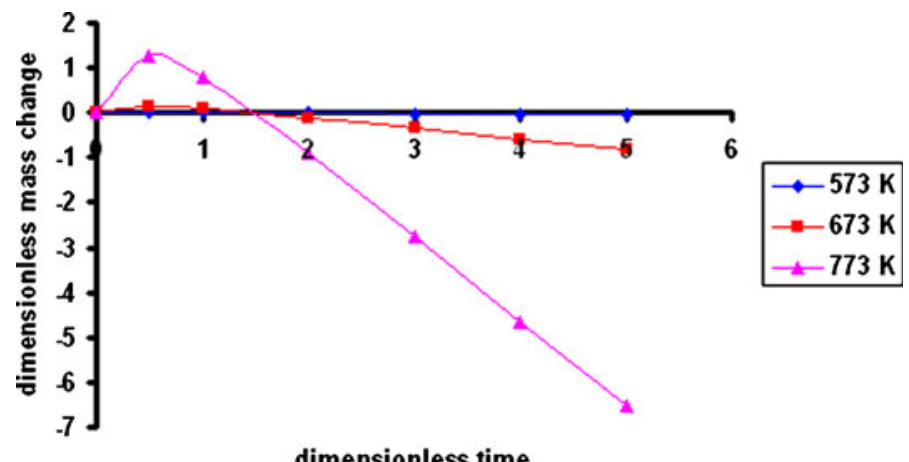

Figure 4. Transient variation of dimensionless mass change at different temperatures, for $\mathrm{NiO}$ (mass fraction of oxide constituent, $\mathrm{f}_{\mathrm{m}}=0.4$ ).

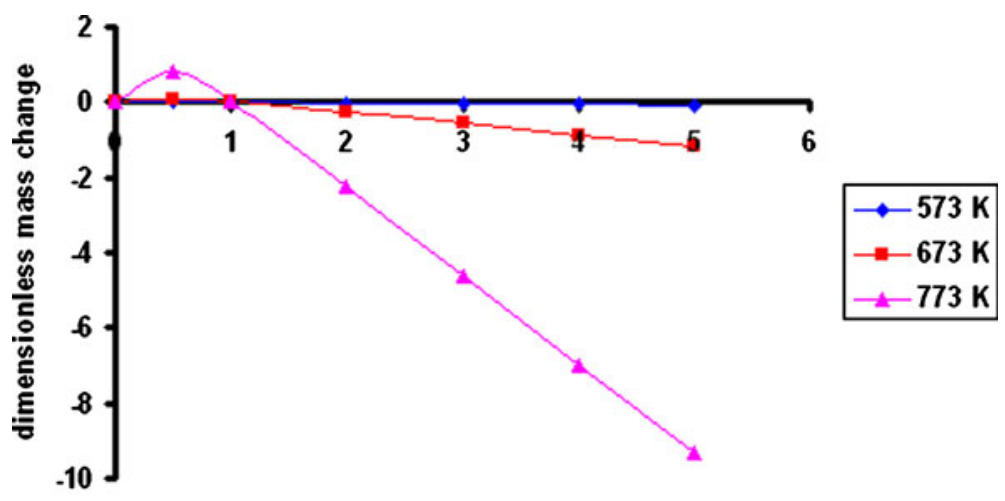

dimensionless time

Figure 5. Transient variation of dimensionless mass change at different temperatures, for $\mathrm{NiO}$ (mass fraction of oxide constituent, $\mathrm{f}_{\mathrm{m}}=0.5$ ).

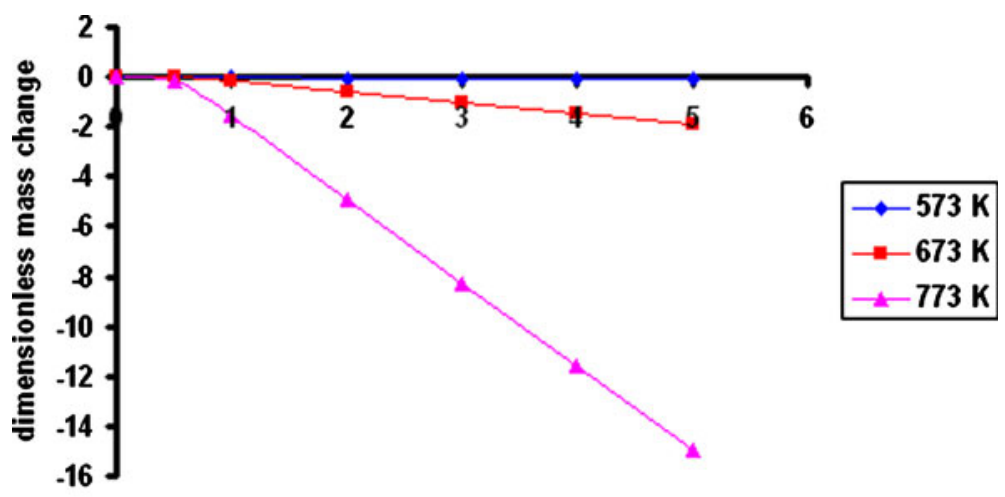

dimensionless time

Figure 6. Transient variation of dimensionless mass change at different temperatures, for $\mathrm{NiO}$ (mass fraction of oxide constituent, $\mathrm{f}_{\mathrm{m}}=0.7$ ). 


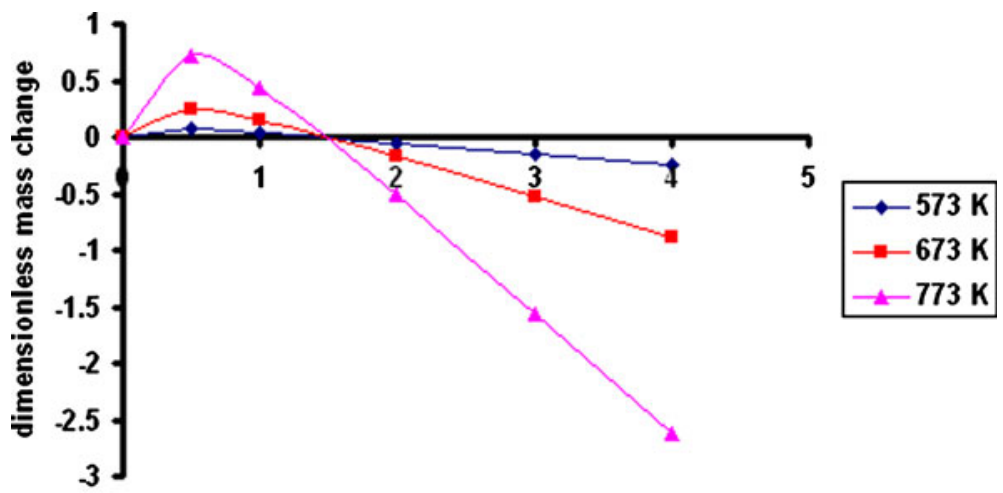

dimensionless time

Figure 7. Transient variation of dimensionless mass change at different temperatures, for $\mathrm{FeO}$ (mass fraction of oxide constituent, $\mathrm{f}_{\mathrm{m}}=0.4$ ).

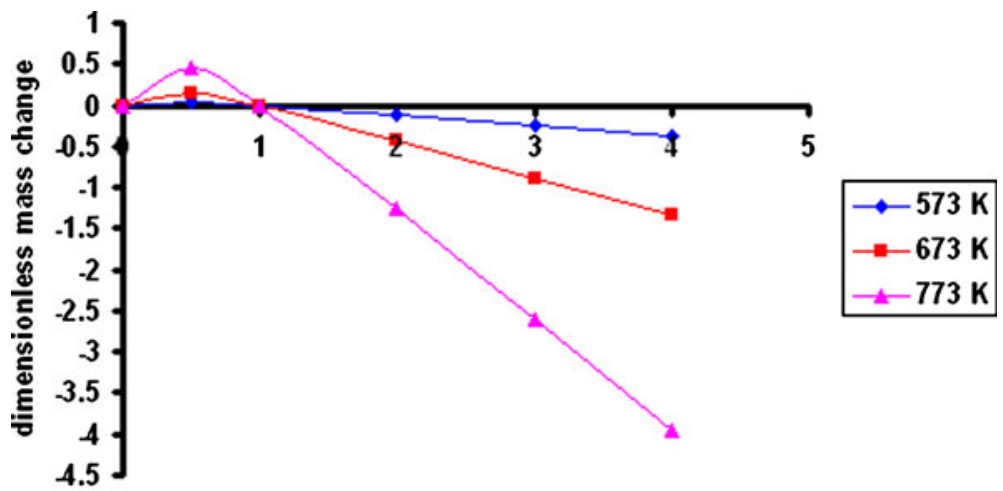

\section{dimensionless time}

Figure 8. Transient variation of dimensionless mass change at different temperatures, for $\mathrm{FeO}$ (mass fraction of oxide constituent, $\mathrm{f}_{\mathrm{m}}=0.5$ ).

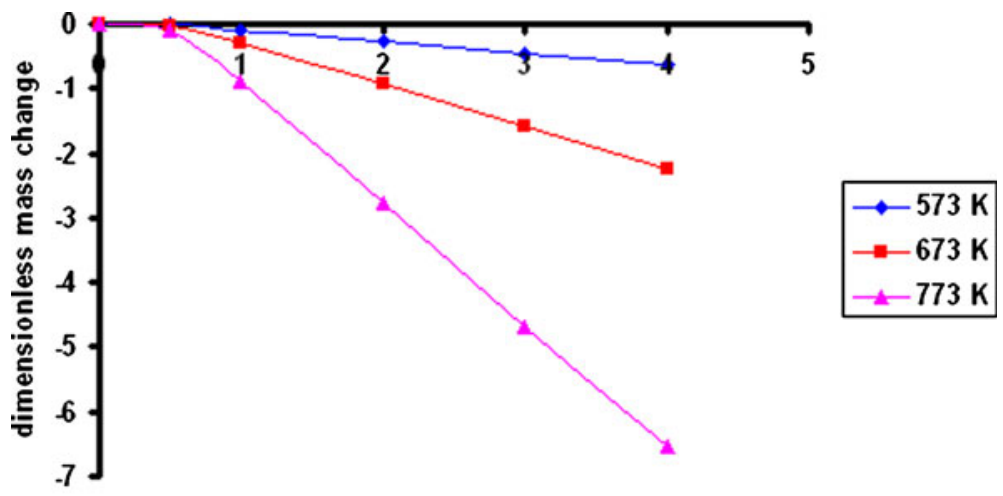

\section{dimensionless time}

Figure 9. Transient variation of dimensionless mass change at different temperatures, for $\mathrm{FeO}$ (mass fraction of oxide constituent, $\mathrm{f}_{\mathrm{m}}=0.7$ ). 


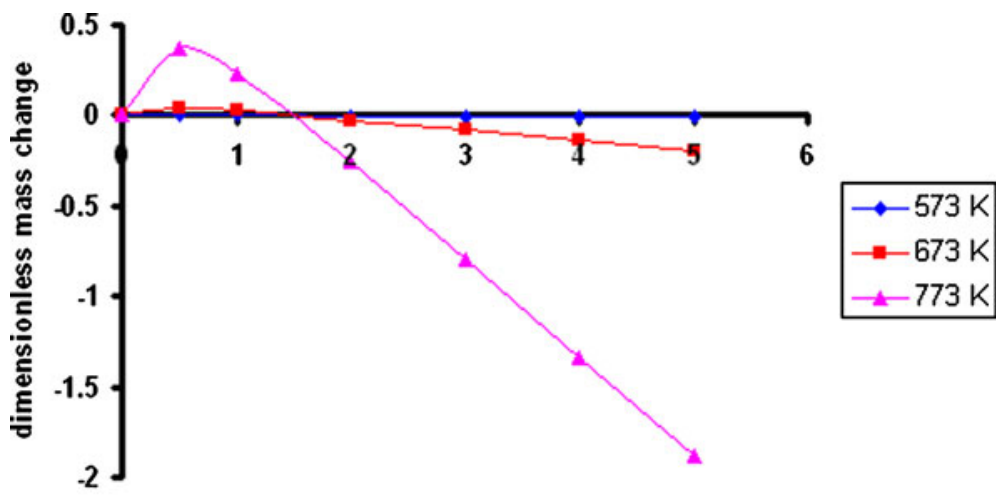

dimensionless time

Figure 10. Transient variation of dimensionless mass change at different temperatures, for $\mathrm{Cr}_{2} \mathrm{O}_{3}$ (mass fraction of oxide constituent, $\mathrm{f}_{\mathrm{m}}=0.4$ ).

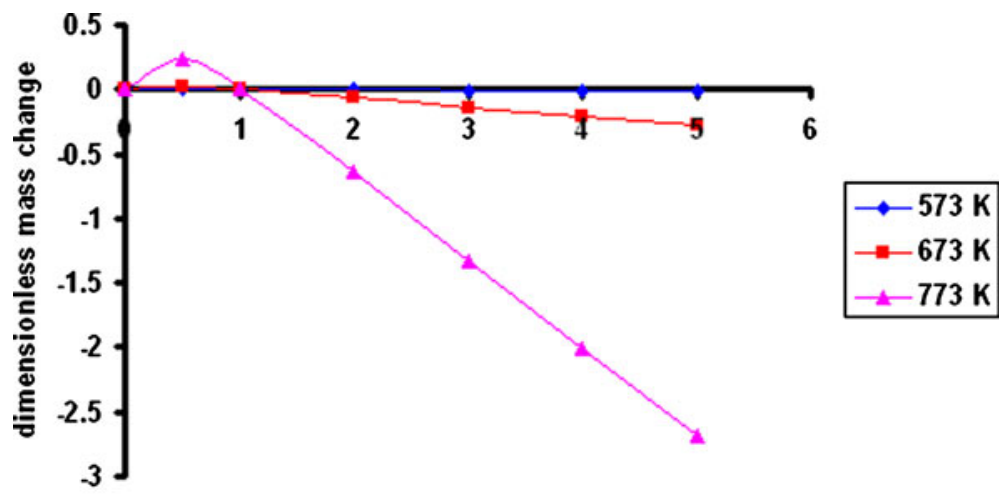

dimensionless time

Figure 11. Transient variation of dimensionless mass change at different temperatures, for $\mathrm{Cr}_{2} \mathrm{O}_{3}$ (mass fraction of oxide constituent, $\mathrm{f}_{\mathrm{m}}=0.5$ ).

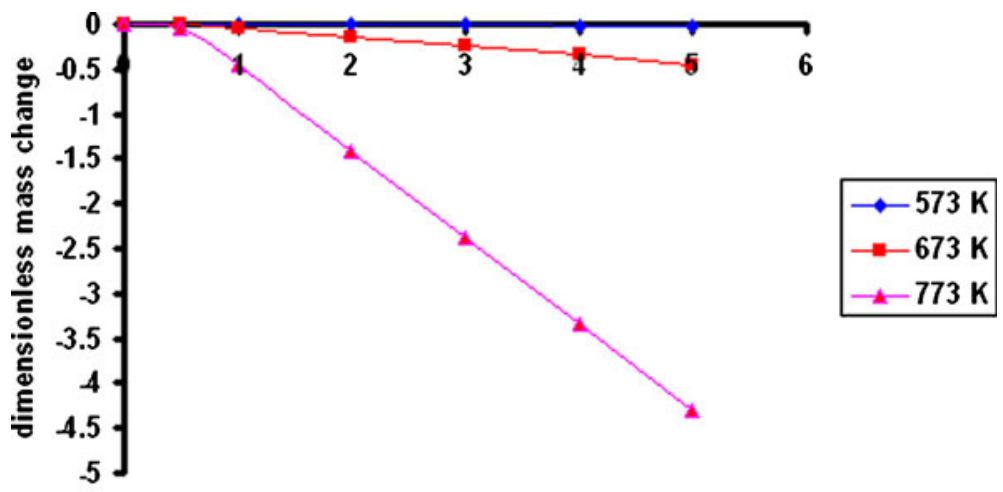

\section{dimensionless time}

Figure 12. Transient variation of dimensionless mass change at different temperatures, for $\mathrm{Cr}_{2} \mathrm{O}_{3}$ (mass fraction of oxide constituent, $f_{m}=0.7$ ). 


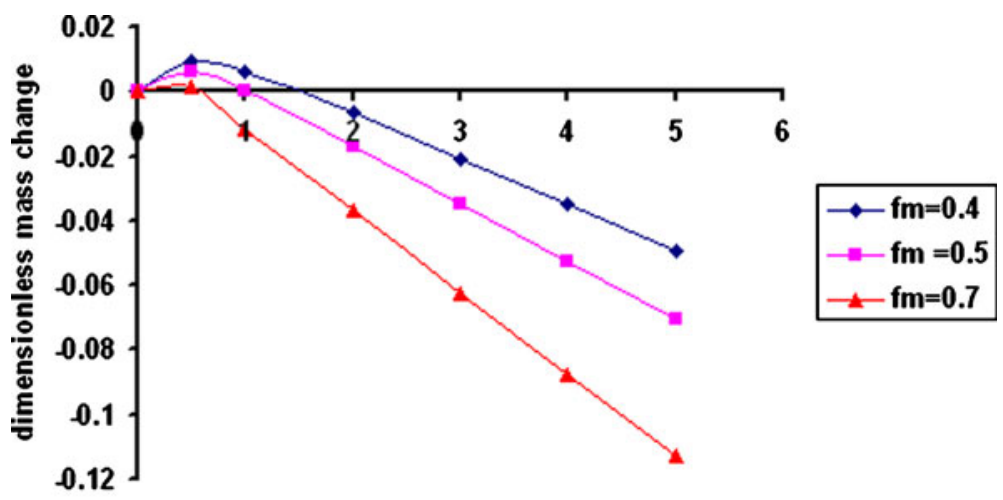

dimensionless time

Figure 13. Transient variation of dimensionless mass change at different mass fraction of oxide constituent for $\mathrm{NiO}$ (temperature $=573 \mathrm{~K}$ ).

increase from zero in an asymptotic manner. It may be observed from the same figure that the scale growth becomes asymptotic and remains invariant beyond a value of 4 on the dimensionless time axis. Figures 4, 5 and 6 show transient variation of erosion-oxidation-induced change of mass per unit area in dimensionless form, $\Delta M^{*}$ (as defined in eqn. 20) for various mass fractions of nickel oxide associated with metallic constituent, i.e., $f_{m}=0.4,0.5$ and 0.7 at different substrate temperatures $573 \mathrm{~K}, 673 \mathrm{~K}$ and $773 \mathrm{~K}$ respectively. Figures 7, 8 and 9 show transient variation of change of mass per unit area in dimensionless form, $\Delta M^{*}$ for various mass fractions of iron oxide associated with metallic constituent, i.e., $f_{m}=0.4,0.5$ and 0.7 at similar substrate temperatures $573 \mathrm{~K}, 673 \mathrm{~K}$ and $773 \mathrm{~K}$ respectively. Figures 10,11 and 12 show transient variation of change of mass per unit area in dimensionless form, $\Delta M^{*}$ for various mass fractions of chromium oxide associated with metallic constituent, i.e., $f_{m}=0.4,0.5$ and 0.7 at different substrate temperatures $573 \mathrm{~K}, 673 \mathrm{~K}$ and $773 \mathrm{~K}$ respectively. It may be observed from the above figures that transient variation of change of mass behaviour is significantly influenced by the temperature of the substrate. With the commencement of oxidation, the initial mass change

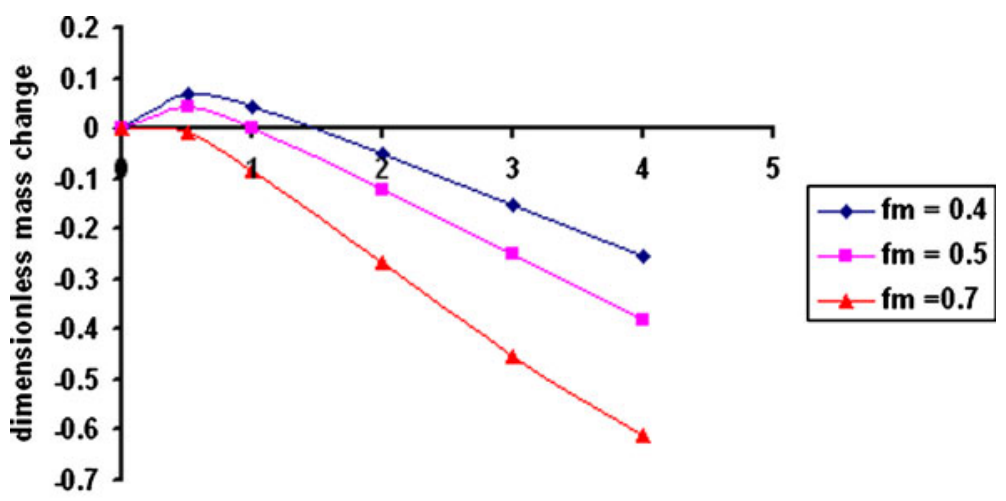

dimensionless time

Figure 14. Transient variation of dimensionless mass change at different mass fraction of oxide constituent for $\mathrm{FeO}$ (temperature $=573 \mathrm{~K}$ ). 


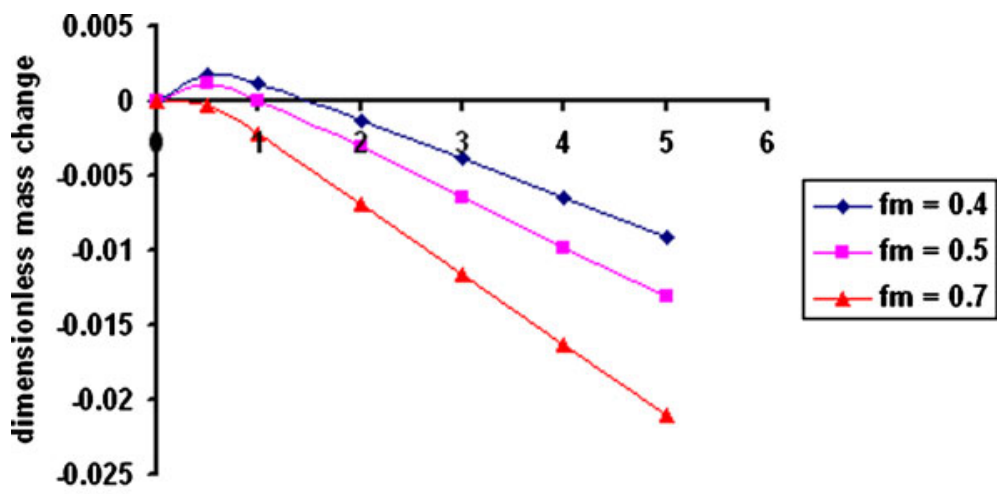

dimensionless time

Figure 15. Transient variation of dimensionless mass change at different mass fraction of oxide constituent for $\mathrm{Cr}_{2} \mathrm{O}_{3}$ (temperature $=573 \mathrm{~K}$ ).

is positive because of the formation of oxide scale. With the advancement of the time, the brittle oxide scale is removed along with subsequent material loss on the substrate. The general nature of the curves shown in figures 4-12 depict an initial increase in mass with time, because of formation of oxide scale and subsequently transitioning to a net loss of mass loss with time at a constant rate. With the increase of the temperature the magnitude of initial mass gain also increases. Further, rate of mass loss also increases as the substrate temperature increases for all values of mass fraction of oxide constituent. It may be inferred that, the higher magnitude of initial mass loss for nickel oxide is attributed to the relative faster rate of growth of the oxide scale in comparison to iron oxide and chromium oxide. This is because the oxidation reaction for formation of nickel oxide requires relatively lower activation energy for initiation of reaction with respect to iron and chromium oxides. As the activation energy associated with the oxidation reaction for chromium is higher; rate of oxidation reaction and scale growth rate are slower with respect to nickel and iron. As a consequence, oxide scale growth is slower which results in lower magnitude of mass loss. The predictions realistically simulate the erosion-oxidation interaction for different oxide scales.

Figures 13, 14 and 15 depict transient variation of mass change $\Delta M^{*}$ at various mass fraction of oxide constituent at substrate temperature, $573 \mathrm{~K}$. It may be observed from these figures that the magnitude of mass change is higher for higher mass fraction of oxide constituent at the given substrate temperature. The characteristics of stochastic modelling based prediction results and their order of magnitude to quantify mass loss resulted from erosion-oxidation interactions are in good agreement with the published literature (Markworth 1992; Wright et al 1995; Stephenson et al 1985, 1986; Stephenson \& Nicholls 1990). Thus, erosion-oxidation simulation code has been satisfactorily validated for characterization of erosion-oxidation interaction phenomena involving multiple/composite oxide scale formation.

\section{Conclusion}

Although some reasonable progress has been reported during the last decade towards developing predictive capability for the service life of metals subjected to high temperature erosionoxidation conditions, advances have been reasonably slow. This is attributed to the inherent 
phenomenological complexity of the coupled kinetics and rudimentary level of knowledge to characterize such complex phenomena. The knowledge of interaction between erosion and oxidation is limited because of the stochastic nature of the phenomena and involvement of large number of variable. Rate of oxide scale growth for nickel is higher with respect to iron and chromium oxides, because of lower activation energy associated with the oxide formation reaction of nickel. In general, this is attributed to the faster rate of reaction linked with lower activation energy. A comparative study has been conducted utilizing the building blocks of Markworth's stochastic model on erosion-oxidation induced damage of boiler grade steel at different temperature with respect to nickel, iron and chromium oxide. It has been observed that, the erosion loss is highest in the case of nickel oxide under boiler operating conditions.

The present investigation of erosion-oxidation interaction analysis on boiler grade steel is expected to be useful to quantify the relative material loss (damage) as a function of time, with respect to three different oxides, namely, nickel, iron and chromium oxides. This can be attributed to the fact that most of the material loss will be resulted as a consequence of shedding of oxide scale rather than by cutting wear (micro-machining action), repeated plastic deformation and abrasion of the metallic substrate. One of the critical variables in this process is the erodent flux striking the target material. Particle kinetic energy required to activate this material loss is expected to be lower, than that required for metal loss by erosion in the absence of any oxidation. Although the stochastic model-based predictions does appear to be physically reasonable to address simple erosion-oxidation phenomena, further improvements can be made with incorporation of more realistic features and pertinent experimental data on the rate parameters of multi-species oxidation kinetics at elevated temperatures which would address simultaneous composite oxide growth phenomena.

\section{References}

Barklow R H and Pettit F S 1979 Corrosion/erosion of materials in coal combustion gas turbines, Proc. Conf. Corrosion/Erosion of Coal Conversion System Materials, Berkeley, USA, Jan 24-26, 1979, NACE, Houston, TX, 139-173

Bitter J G 1963a A study of erosion phenomenon, Wear 6: (Part 1) 5-21

Bitter J G 1963b A study of erosion phenomenon, Wear 6: (Part 2) 169-191

Chang S L, Pettit F S and Birks N 1990 Oxidation of Metals 34: 71-100

Finnie I 1960 Erosion of surfaces by solid particles, Wear 3: 87-103

Hogmark S, Hammarsten A and Soderberg S 1983 Proceedings of the Sixth International Conference on Erosion by Solid and Liquid Impact: $37-48$

Markworth A J 1992 A stochastic model for the simultaneous occurrence of oxidation and erosion, Mater. Sci. Eng. A 150: 37-41

Nicholls J R and Stephenson D J 1995 Monte-Carlo modelling of erosion processes, Wear 186-187: 64-77

Shewmon P G 1981 Particle size threshold in the erosion of metals, Wear 68: 253-258

Stack M M and Pena D 2001 Mapping erosion of Ni-Cr/WC-based composites at elevated temperatures: some recent advances, Wear 251: 1433-1443

Stack M M and Scott F H 1993 The role of oxides in the erosion-corrosion of alloys under low velocity conditions, Corrosion Sci. 35: 1027-1040

Stephenson D J and Nicholls J R 1990 Role of surface oxides in modifying solid particle impact damage, J. Mater. Sci. Technol. 6: 96-99

Stephenson D J, Nicholls J R and Hancock P 1985 The erosion of gas turbine blade materials by solid sea salt, Corrosion Science 25: 1181-1192 
Stephenson D J, Nicholls J R and Hancock P 1986 High temperature erosion-oxidation mechanism, maps and models, Wear 111: 15-29, 31-39

Wellman R G and Nicholls J R 2004 A Monte-Carlo model for predicting the erosion rate of EB PVD TBCs, Wear 256: 889-899

Wright I G, Sethi V K and Markworth A J 1995 A generalized description of the simultaneous processes of scale growth by high-temperature oxidation and removal by erosive impact, Wear 186: 230-237 